\title{
Charm quark transport within viscous QCD medium : colliding and radiating
}

\author{
Adiba Shaikh, ${ }^{a, *}$ Manu Kurian, ${ }^{b}$ Santosh K. Das, ${ }^{c}$ Vinod Chandra, ${ }^{b}$ Sadhana Dash ${ }^{a}$ \\ and Basanta K. Nandi ${ }^{a}$ \\ ${ }^{a}$ Department of Physics, Indian Institute of Technology Bombay, \\ Powai, Mumbai-400076, India \\ ${ }^{b}$ Indian Institute of Technology Gandhinagar, \\ Gandhinagar-382355, Gujarat, India \\ ${ }^{c}$ School of Physical Sciences, Indian Institute of Technology Goa, \\ Ponda-403401, Goa, India \\ E-mail: adibashaikh9@gmail.com, manu.kurian@iitgn.ac.in
}

We study the momentum and temperature dependence of the charm quark transport coefficients, i.e., drag and momentum diffusion for collisional and radiative processes in a viscous QCD medium. The thermal medium interactions are included through the effective fugacity quasiparticle model, which modifies the distribution function of the QGP particles by the introduction of temperature-dependent effective fugacity parameter. Shear viscous corrections to the charm quark transport coefficients are incorporated at leading order through the near-equilibrium distribution function by solving the effective Boltzmann equation within relaxation time approximation. Preliminary results for bulk viscous corrections are also presented within the same framework.

\footnotetext{
*** 10th International Workshop on Charm Physics (CHARM2020), ***

*** 31 May - 4 June, 2021 ***

*** Mexico City, Mexico - Online ***
}

\footnotetext{
${ }^{*}$ Speaker
} 


\section{Introduction}

Quark-gluon plasma (QGP) is a deconfined phase of QCD with quarks and gluons as effective degrees of freedom. This phase of matter exists at high temperature and/or high baryon density, e.g., in the heavy-ion collision experiments such as Relativistic Heavy Ion Collider (RHIC) and Large Hadron Collider (LHC). Heavy quarks, like charm and bottom, are created mostly in the early stage of the collision through the hard scattering process. Due to their large mass $\left(m_{H Q}\right)$ relative to the QGP temperature $(T)$, they do not thermalize with the fireball and acts as an effective probe to study the QGP properties [1-5]. Heavy quark traversing through the QCD medium suffers energy loss due to collision with the plasma constituents and radiation of the soft gluons. Collisional energy loss is dominant for the heavy quark with low momentum and at high momentum medium induced gluon radiation becomes dominant. Within Fokker-Planck dynamics, one can treat heavy quarks to be executing Brownian motion within the plasma where the interactions of the heavy quarks with the medium constituents are incorporated through the drag and momentum diffusion coefficients [6-8]. The heavy quark transport coefficients are sensitive to the medium evolution in presence of the dissipative processes within QGP. The transport coefficients in the hot and dense QCD medium can be determined from the underlying microscopic theories such as the effective kinetic theory approach. They could also be extracted experimentally through various observables at RHIC and LHC. The viscous corrections to the heavy quark transport coefficients due to the collisional processes have been previously studied [9-13].

We study the sensitivity of the heavy quark drag and diffusion coefficients to the shear and bulk viscosities of QGP for the radiative process. The realistic equation of state effects is incorporated into the analysis through the effective fugacity quasiparticle model (EQPM) description of the QCD medium [14, 15]. The non-equilibrium distribution function has been obtained by solving the consistently developed effective Boltzmann equation based on the EQPM by employing the Chapman-Enskog like iterative method within the relaxation time approximation (RTA) [16]. The mean-field contributions originating from the basic conservation laws are incorporated in the estimation of the near-equilibrium momentum distribution functions. Here, we present the result for the effect of shear and bulk viscous corrections to the radiative process of the heavy quark in terms of its transport coefficients and compare it to the collisional process. We observe a considerable modification in the drag and diffusion coefficients of the heavy quark due to these corrections.

In section 2, the formulation of the heavy quark transport coefficients is discussed for collisional and radiative processes followed by the EQPM description of viscous corrections to the momentum distribution function of quasiparticles. Section 3 focuses on the results for drag and diffusion coefficients of the charm quark including shear and bulk viscous corrections. We summarize with the conclusion in section 4 . 


\section{Formalism}

\subsection{Heavy quark transport coefficients for collision and radiative processes}

The heavy quark $(H Q)$ undergoes collisions with the medium constituents, i.e., light quarks $(l q)$, light antiquarks $(\bar{l} q)$ and gluons $(g)$. The elastic $(2 \rightarrow 2)$ process is,

$$
H Q(p)+l q / l \bar{q} / g(q) \rightarrow H Q\left(p^{\prime}\right)+l q / l \bar{q} / g\left(q^{\prime}\right) .
$$

Considering soft scattering approximation, the Boltzmann transport equation for the evolution of the heavy quark momentum distribution $\left(f_{H Q}\right)$ reduces to the Fokker-Planck equation [6],

$$
\frac{\partial f_{H Q}}{\partial t}=\frac{\partial}{\partial p_{i}}\left[A_{i}(\mathbf{p}) f_{H Q}+\frac{\partial}{\partial p_{j}}\left(B_{i j}(\mathbf{p}) f_{H Q}\right)\right]
$$

where $A_{i}(\mathbf{p})$ corresponds to the drag force acting on the heavy quark given as,

$$
\begin{aligned}
A_{i}(\mathbf{p})= & \frac{1}{2 E_{p} \gamma_{H Q}} \int \frac{d^{3} \mathbf{q}}{(2 \pi)^{3} E_{q}} \int \frac{d^{3} \mathbf{q}^{\prime}}{(2 \pi)^{3} E_{q^{\prime}}} \int \frac{d^{3} \mathbf{p}^{\prime}}{(2 \pi)^{3} E_{p^{\prime}}} \times \sum\left|\mathcal{M}_{2 \rightarrow 2}\right|^{2}(2 \pi)^{4} \\
& \times \delta^{(4)}\left(p+q-p^{\prime}-q^{\prime}\right) f_{k}\left(E_{q}\right)\left(1 \pm f_{k}\left(E_{q^{\prime}}\right)\right)\left[\left(p-p^{\prime}\right)_{i}\right]=\left\langle\left\langle\left(p-p^{\prime}\right)_{i}\right\rangle\right\rangle \\
= & p_{i} A\left(p^{2}\right),
\end{aligned}
$$

and $B_{i j}(\mathbf{p})$ gives the diffusion in momentum space expressed in the above form of thermal average as,

$$
B_{i j}(\mathbf{p})=\frac{1}{2}\left\langle\left\langle\left(p-p^{\prime}\right)_{i}\left(p-p^{\prime}\right)_{j}\right\rangle\right\rangle=\left[\delta_{i j}-\frac{p_{i} p_{j}}{p^{2}}\right] B_{0}\left(p^{2}\right)+\frac{p_{i} p_{j}}{p^{2}} B_{1}\left(p^{2}\right),
$$

where $\gamma_{H Q}=N_{s} \times N_{c}$ is the heavy quark degeneracy factor with $N_{s}=2, N_{f}=3(u, d, s)$, and $N_{c}=3$ (for $S U(3)$ ). $\left|\mathcal{M}_{2 \rightarrow 2}\right|$ represents the scattering amplitude for $2 \rightarrow 2$ process explicitly specified in the Appendix $\mathbf{A}$ of ref. [17] and $p=|\mathbf{p}|$ is the magnitude of heavy quark initial momentum. $f_{k}\left(E_{q}\right)$ denotes the distribution function for quarks, antiquarks $(k=l q, l \bar{q})$ and gluons $(k=g)$. We have also incorporated the Fermi suppression $\left(1-f_{l q}\left(E_{q^{\prime}}\right)\right)$ and Bose enhancement $\left(1+f_{g}\left(E_{q^{\prime}}\right)\right)$ factors for the final state phase space of the light quarks and gluons, respectively.

From Eq.(2), the heavy quark drag coefficient $(A)$, transverse diffusion $\left(B_{0}\right)$ and longitudinal diffusion $\left(B_{1}\right)$ coefficients are defined as,

$$
\begin{aligned}
& A=\langle\langle 1\rangle\rangle-\frac{\left\langle\left\langle\mathbf{p} \cdot \mathbf{p}^{\prime}\right\rangle\right\rangle}{p^{2}}, \\
& B_{0}=\frac{1}{4}\left[\left\langle\left\langle p^{\prime 2}\right\rangle\right\rangle-\frac{\left\langle\left\langle\left(\mathbf{p} \cdot \mathbf{p}^{\prime}\right)^{2}\right\rangle\right\rangle}{p^{2}}\right], \\
& B_{1}=\frac{1}{2}\left[\frac{\left\langle\left\langle\left(\mathbf{p} \cdot \mathbf{p}^{\prime}\right)^{2}\right\rangle\right\rangle}{p^{2}}-2\left\langle\left\langle\left(\mathbf{p} \cdot \mathbf{p}^{\prime}\right)\right\rangle\right\rangle+p^{2}\langle\langle 1\rangle\rangle\right],
\end{aligned}
$$


In the centre-of-momentum frame of the system, the thermal average of a function $F(\mathbf{p})$, in general, for $2 \rightarrow 2$ process becomes,

$$
\begin{aligned}
\langle\langle F(\mathbf{p})\rangle\rangle_{c o l}= & \frac{1}{\left(512 \pi^{4}\right) E_{p} \gamma_{H Q}} \int_{0}^{\infty} d q\left(\frac{s-m_{H Q}^{2}}{s}\right) f_{k}\left(E_{q}\right)\left(1 \pm f_{k}\left(E_{q^{\prime}}\right)\right) \int_{0}^{\pi} d \chi \sin \chi \\
& \times \int_{0}^{\pi} d \theta_{c m} \sin \theta_{c m} \sum\left|\mathcal{M}_{2 \rightarrow 2}\right|^{2} \int_{0}^{2 \pi} d \phi_{c m} F(\mathbf{p}),
\end{aligned}
$$

where $\chi$ is the angle between the heavy quark and medium particles in the lab frame and $s=\left(E_{p}+E_{q}\right)^{2}-|\mathbf{p}|^{2}-|\mathbf{q}|^{2}-2|\mathbf{p}||\mathbf{q}| \sin \chi$. The zenith $\theta_{c m}$ and azimuthal $\phi_{c m}$ angles are defined in the center-of-momentum frame. The Debye screening mass $\left(m_{D}\right)$ is inserted at leading order for $t$-channel gluonic propagator to the in-medium matrix elements.

Along with collision, the heavy quarks can also radiate soft gluons induced by the QGP medium. The inelastic $(2 \rightarrow 3)$ process is,

$$
H Q(p)+l q / l \bar{q} / g(q) \rightarrow H Q\left(p^{\prime}\right)+l q / l \bar{q} / g\left(q^{\prime}\right)+g\left(k^{\prime}\right),
$$

where $k^{\prime} \equiv\left(E_{k^{\prime}}, \mathbf{k}_{\perp}^{\prime}, k_{z}^{\prime}\right)$ is the four-momentum of the final state soft gluon $\left(k^{\prime} \rightarrow 0\right)$ emitted by the heavy quark. Comparing this process with the collision case, only the kinematical and the interaction parts are modified in Eq. (7). The general expression for the thermal averaged $F$ (p) for $2 \rightarrow 3$ process is [18],

$$
\begin{aligned}
\langle\langle F(\mathbf{p})\rangle\rangle_{\text {rad }}= & \langle\langle F(\mathbf{p})\rangle\rangle_{c o l} \times \int \frac{d^{3} \mathbf{k}^{\prime}}{(2 \pi)^{3} E_{k^{\prime}}} \sum\left|\mathcal{M}_{2 \rightarrow 3}\right|^{2} \delta^{(4)}\left(p+q-p^{\prime}-q^{\prime}-k^{\prime}\right) \\
& \times\left(1+f_{k}\left(E_{k^{\prime}}\right)\right) \theta_{1}\left(E_{p}-E_{k^{\prime}}\right) \theta_{2}\left(\tau-\tau_{F}\right),
\end{aligned}
$$

where the theta function $\theta_{1}\left(E_{p}-E_{k^{\prime}}\right)$ allows the initial heavy quark energy $E_{p}$ to be greater than the radiated soft gluon energy $E_{k^{\prime}}$ in the final state and $\theta_{2}\left(\tau-\tau_{F}\right)$ ensures that the collision time $\tau$ of the heavy quark with the medium particles is greater than the gluon formation time $\tau_{F}$ (LPM Effect) [19]. $\left(1+f_{g}\left(E_{k^{\prime}}\right)\right)$ is the Bose enhancement factor for the radiated gluon and $\left|\mathcal{M}_{2 \rightarrow 3}\right|^{2}$ is the matrix element squared for the radiative process, which can be written as [20],

$$
\left|\mathcal{M}_{2 \rightarrow 3}\right|^{2}=\left|\mathcal{M}_{2 \rightarrow 2}\right|^{2} \times \frac{12 g_{s}^{2}}{\left(k_{\perp}^{\prime}\right)^{2}}\left(1+\frac{m_{H Q}^{2}}{s} e^{2 y_{k^{\prime}}}\right)^{-2},
$$

where $y_{k^{\prime}}$ is the rapidity of the emitted soft gluon and $\left(1+\frac{m_{H Q}^{2}}{s} e^{2 y_{k^{\prime}}}\right)^{-2}$ is the dead cone factor for the heavy quark. The evaluation of the soft gluon 3-momentum integral is discussed in detail in Appendix B of ref. [17]. 


\subsection{EQPM distribution function for a viscous QCD medium}

The realistic hot QCD medium equation of state can be interpreted in terms of non-interacting quasiparticles having temperature-dependent effective fugacities. The effects of the thermal medium interactions are included through the EQPM based on the lattice QCD equation of state $[14,15]$. For the system close to the local equilibrium, the momentum distribution function of the medium particles takes the form,

$$
f_{k}=f_{k}^{0}+\delta f_{k} \quad \text { with } \delta f_{k} / f_{k}^{0} \ll 1,
$$

where $f_{k}^{0}$ is the EQPM equilibrium distribution function $\delta f_{k}$ is the non-equilibrium component. The EQPM distribution functions for the light quarks/antiquarks and gluons is defined in terms of effective fugacity parameter $z_{k}$ which encodes the QCD medium interactions as follows (for vanishing baryon chemical potential),

$$
f_{l q / l \bar{q}}^{0}=\frac{z_{l q / l \bar{q}} \exp [-\beta(u \cdot q)]}{1+z_{l q / l \bar{q}} \exp [-\beta(u \cdot q)]}, \quad f_{g}^{0}=\frac{z_{g} \exp [-\beta(u \cdot q)]}{1-z_{g} \exp [-\beta(u \cdot q)]} .
$$

The parameters $z_{l q / l \bar{q}}$ and $z_{g}$ are effective fugacities that encode the QCD interactions for quarks/antiquarks and gluons. These temperature-dependent parameters modify the single-particle dispersion relation as,

$$
{\tilde{q_{k}}}^{\mu}=q_{k}^{\mu}+\delta \omega_{k} u^{\mu}, \quad \delta \omega_{k}=T^{2} \partial_{T} \ln \left(z_{k}\right),
$$

where $\tilde{q}_{k}^{\mu}=\left(\omega_{k}, \mathbf{q}_{k}\right)$ and $q_{k}^{\mu}=\left(E_{q}, \mathbf{q}_{k}\right)$ are the quasiparticle and bare particle momenta, respectively, $u^{\mu}$ is the normalized fluid velocity such that $u^{\mu} u_{\mu}=1$ with metric tensor $g^{\mu \nu}=$ $\operatorname{diag}(1,-1,-1,-1)$, and ${\tilde{q_{k}}}^{0} \equiv \omega_{k}=E_{q}+\delta \omega_{k}$, is the quasiparticle energy. The effective strong coupling constant $\left(\alpha_{e f f}\right)$ is introduced through EQPM based Debye mass [21].

Using the effective covariant kinetic theory approach, the evolution of the distribution function is described by the effective Boltzmann transport equation based on the EQPM. Within RTA, it is has a form [16],

$$
\tilde{q}_{k}^{\mu} \partial_{\mu} f_{k}\left(x, \tilde{q}_{k}\right)+F_{k}^{\mu}\left(u \cdot \tilde{q}_{k}\right) \partial_{\mu}^{(q)} f_{k}=-\left(u \cdot \tilde{q}_{k}\right) \frac{\delta f_{k}}{\tau_{R}},
$$

where $\tau_{R}$ is the thermal relaxation time and $F_{k}^{\mu}=-\partial_{v}\left(\delta \omega_{k} u^{v} u^{\mu}\right)$ correspond to the mean-field force term. The viscous corrections to the distribution function are obtained by solving Eq. (13) by using iterative Chapman-Enskog like method [22] to obtain the leading order correction to the distribution function as,

$$
\delta f_{k}=\tau_{R}\left(\tilde{q}_{k}^{\gamma} \partial_{\gamma} \beta+\frac{\beta \tilde{q}_{k}^{\gamma} \tilde{q}_{k}^{\phi}}{u \cdot \tilde{q}_{k}} \partial_{\gamma} u_{\phi}-\beta \theta \delta \omega_{k}\right) f_{k}^{0} \tilde{f}_{k}^{0},
$$

where $\tilde{f}_{k}^{0} \equiv\left(1-a_{k} f_{k}^{0}\right)\left(a_{g}=-1\right.$ for bosons and $a_{l q}=+1$ for fermions $)$.

The first-order evolution for the shear stress tensor $\pi^{\mu v}$ and bulk viscous pressure $\Pi$ within the effective kinetic theory has the following forms,

$$
\pi^{\mu v}=2 \tau_{R} \beta_{\pi} \sigma^{\mu v}, \quad \Pi=-\tau_{R} \beta_{\Pi} \theta,
$$


with $\theta \equiv \partial_{\mu} u^{\mu}$ as the scalar expansion and $\sigma^{\mu \nu} \equiv \Delta_{\alpha \beta}^{\mu \nu} \nabla^{\alpha} u^{\beta}$ where $\Delta_{\alpha \beta}^{\mu \nu} \equiv \frac{1}{2}\left(\Delta_{\alpha}^{\mu} \Delta_{\beta}^{v}+\Delta_{\beta}^{\mu} \Delta_{\alpha}^{v}\right)-$ $\frac{1}{3} \Delta^{\mu \nu} \Delta_{\alpha \beta}$ denotes traceless symmetric projection operator orthogonal to the fluid velocity $u^{\mu}$. Here, $\beta_{\pi}$ and $\beta_{\Pi}$ are the first-order coefficients [23].

Using the evolution equation, the shear and bulk viscous corrections to the distribution function can be expressed as,

$$
\begin{aligned}
\delta f_{k} & =\delta f_{k}^{\text {shear }}+\delta f_{k}^{\text {bulk }}, \\
\delta f_{k}^{\text {shear }} & =\frac{\beta f_{k}^{0} \tilde{f}_{k}^{0}}{2 \beta_{\pi}\left(u \cdot \tilde{q}_{k}\right)} \tilde{q}_{k}^{\alpha} \tilde{q}_{k}^{\beta} \pi_{\alpha \beta}, \\
\delta f_{k}^{\text {bulk }} & =-\frac{\beta f_{k}^{0} \tilde{f}_{k}^{0}}{\beta_{\Pi}\left(u \cdot \tilde{q}_{k}\right)}\left[\left(u \cdot \tilde{q}_{k}\right)^{2} c_{s}^{2}-\frac{\left|\tilde{\mathbf{q}}_{k}\right|^{2}}{3}-\left(u \cdot \tilde{q}_{k}\right) \delta \omega_{k}\right] \Pi .
\end{aligned}
$$

Substituting the modified in-medium particle distribution functions with the shear and bulk viscous corrections from Eq. (17) and (18) into Eqs. (4)-(6), we obtain the non-equilibrium corrections to the heavy quark drag and diffusion coefficients in the viscous medium up to first order. Considering longitudinal boost invariant expansion through Bjorken prescription [24] with Milne coordinates $\left(\tau, x, y, \eta_{s}\right)$, the Eq. (17) and Eq. (18) gets simplified to,

$$
\begin{aligned}
\delta f_{k}^{\text {shear }} & =\frac{f_{k}^{0} \tilde{f}_{k}^{0} \mathfrak{s}}{\beta_{\pi} \omega_{k} T \tau}\left(\frac{\eta}{\mathfrak{s}}\right)\left[\frac{\left|\mathbf{q}_{\mathbf{k}}\right|^{2}}{3}-\left(q_{k}\right)_{z}^{2}\right], \\
\delta f_{k}^{\text {bulk }} & =\frac{f_{k}^{0} \tilde{f}_{k}^{0} \mathfrak{s}}{\beta_{\Pi} \omega_{k} T \tau}\left(\frac{\zeta}{\mathfrak{s}}\right)\left[\left(\omega_{k}\right)^{2} c_{s}^{2}-\frac{\left|\mathbf{q}_{\mathbf{k}}\right|^{2}}{3}-\left(\omega_{k}\right) \delta \omega_{k}\right],
\end{aligned}
$$

where $\tau=\sqrt{t^{2}-z^{2}}$ is the proper time and $\eta_{s}=\tanh ^{-1}(z / t)$ is the space-time rapidity with $u^{\mu}=(1,0,0,0)$ and $g^{\mu \nu}=\left(1,-1,-1,-1 / \tau^{2}\right)$. Here, $\theta=1 / \tau, \Pi=-\zeta / \tau$ and $\pi^{\mu \nu} \sigma_{\mu \nu}=4 \eta / 3 \tau^{2}$. $\eta$ and $\zeta$ denotes the shear viscosity and bulk viscosity of the QGP respectively, $c_{s}^{2}$ is the speed of sound squared and $\mathfrak{s}$ is the entropy density in the medium.

\section{Results}

In our analysis, we consider the charm quark mass $m_{c}=1.3 \mathrm{GeV}$, quark-hadron transition temperature $T_{c}=170 \mathrm{MeV}$ for three massless light quark flavors with zero net baryon density and the proper time $\tau=0.25 \mathrm{fm}$. Fig. 1 (upper panel) displays the effect of shear viscous correction on the momentum dependence of the charm quark transport coefficients for collisional and radiative processes. The transport coefficients are scaled with their respective values for $\eta=0$ case. The trend of the plots can be described from Eqs. (4)- (8). It is observed that the shear viscosity reduces the heavy quark drag $(A(\eta) / A(\eta=0))$ (upper-left panel) and is more prominent at low momenta of the charm quark ( $p \approx 1-3 \mathrm{GeV}$ ) wherein the increase of shear viscosity results in a decrease in the drag coefficient for both collisional and radiative processes. However, at high momentum $(p \approx 10 \mathrm{GeV}$ ), the drag coefficient increases with an increase in the shear viscosity to entropy density ratio $\eta / \mathfrak{s}$. This could be realized from the interplay of two terms in Eq. (4) for the low and high momentum regimes while incorporating the viscous effects through Eq. (19). In contrast to 

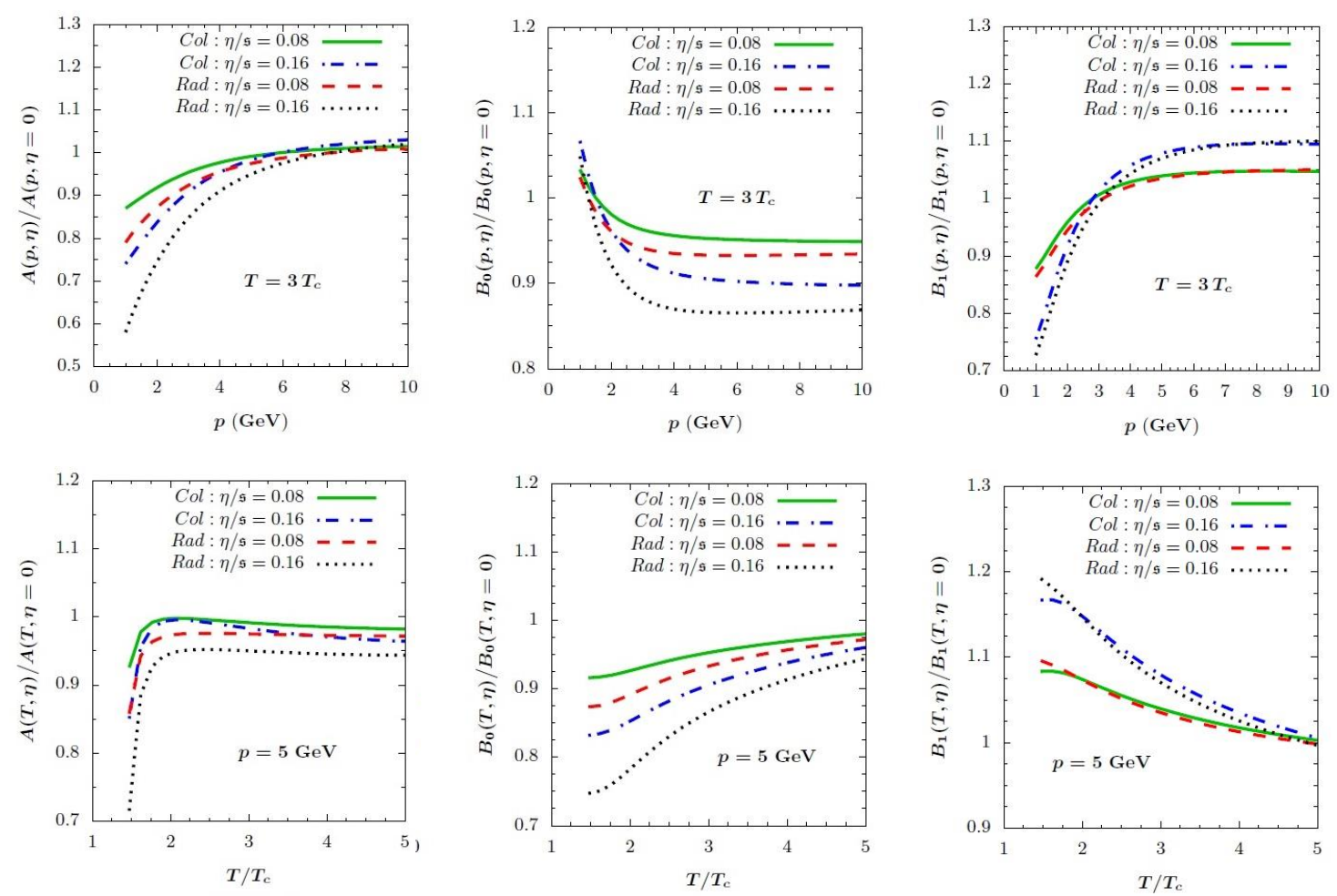

Figure 1: Charm quark transport coefficients with shear viscous correction scaled with corresponding value for non-viscous case $(\eta=0)$ as a function of its initial momentum $p$ (upper panel) at and as a function of QGP temperature $T / T_{c}$ (lower panel).

the drag coefficient, the transverse diffusion coefficient $\left(B_{0}(\eta) / B_{0}(\eta=0)\right)$ (upper-middle panel) increases with the shear viscous correction near $p \approx 1 \mathrm{GeV}$ which further gets suppressed after $p \gtrsim 3 \mathrm{GeV}$. The longitudinal momentum diffusion coefficient $\left(B_{1}(\eta) / B_{1}(\eta=0)\right)$ (upper-right panel) of the charm quark is considerably reduced at low momenta ( $p \lesssim 2 \mathrm{GeV}$ ) affecting both collisional and radiative curves equally with the variation of $\eta / \mathfrak{s}$. For momenta $p \gtrsim 6 \mathrm{GeV}$, the longitudinal diffusion coefficient increases compared to its value in the absence of shear viscosity and higher $\eta / \mathfrak{s}$ results in a larger deviation.

Fig. 1 (lower-left panel) shows the effect of variation of the scaled drag coefficient $A(\eta) / A(\eta=$ 0 ) as a function of the scaled QGP temperature $T / T_{c}$. It is seen that the shear viscous effect reduces the charm quark drag coefficient throughout the considered temperature range at $p=5$ $\mathrm{GeV}$. This behaviour can be accounted by the negative contribution from the factor $\left[\frac{\left|\mathbf{q}_{\mathbf{k}}\right|^{2}}{3}-\left(q_{k}\right)_{z}^{2}\right]$ in Eq. (19) for $\delta f_{k}^{\text {shear }}$. The shear viscous effect is more pronounced near the transition temperature $\left(T \approx 1.5 T_{c}\right.$ ) as observed from the temperature dependence of $\beta_{\pi}$, i.e., $\beta_{\pi} \propto T^{4}$ such that $\frac{\mathfrak{s}}{\beta_{\pi} T} \propto \frac{1}{T^{2}}$ in the definition of $\delta f_{k}^{\text {shear }}$ in Eq. (19). The temperature dependence indicate suppression of the transverse diffusion coefficient $B_{0}(\eta) / B_{0}(\eta=0)$ (lower-middle panel) of the charm quark with an increase in shear viscosity. This suppression, however, is observed to be relatively more for the radiative process compared to the collision. The longitudinal momentum diffusion $\left(B_{1}(\eta) / B_{1}(\eta=0)\right)$ (lower-right panel) seems to increase with an increase in $\eta / \mathfrak{s}$ for both collision 

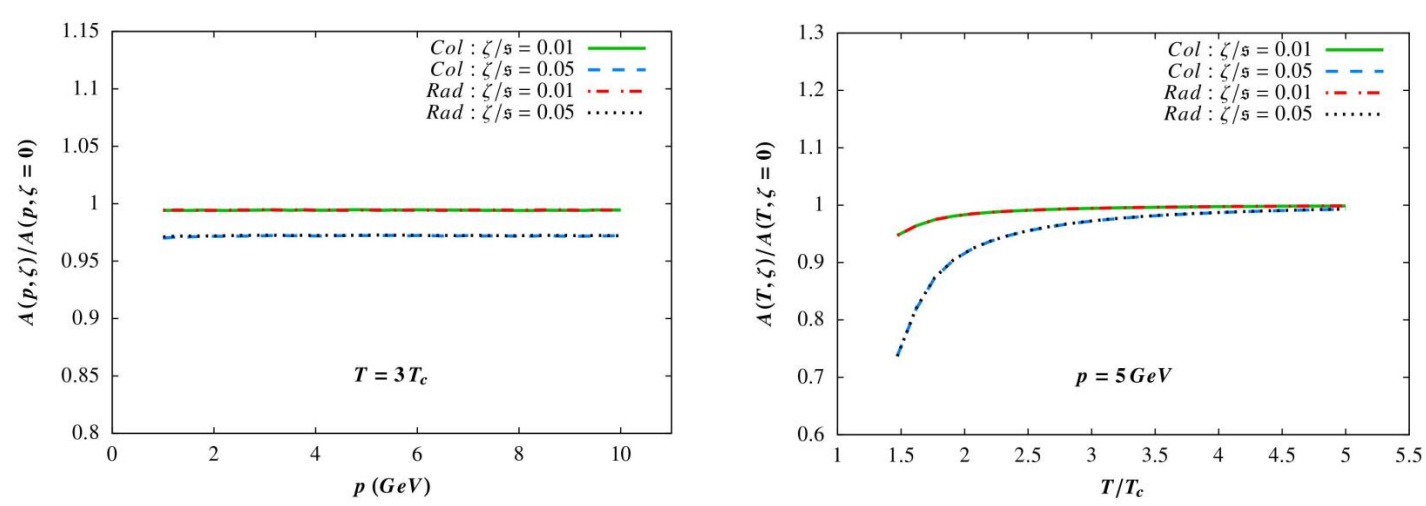

Figure 2: Charm quark transport coefficients with bulk viscous correction scaled with corresponding value for non-viscous case $(\zeta=0)$ as a function of its initial momentum $p$ (left panel) at and as a function of temperature $T / T_{c}$ (right panel).

and radiative processes for $T<4 T_{c}$. The shear viscous effect to the diffusion coefficients is more visible near the low temperature regimes.

In Fig. 2 (left panel), the effect of bulk viscous correction to the momentum dependence of the charm quark drag coefficient $(A(\zeta) / A(\zeta=0))$ is shown. The bulk viscosity seems to reduce the heavy quark drag with a constant dependence throughout the considered momentum range. The drag coefficient ratio decreases with an increase in bulk viscosity to entropy density ratio $\zeta / \mathfrak{s}$. This can be understood by the suppression of $\delta f_{k}^{\text {bulk }}$ due to the negative terms in Eq. (20) with an increase in $\zeta / \mathfrak{s}$. Fig. 2 (right panel) shows the effect of the variation of the scaled drag coefficient as a function of the scaled QGP temperature $T / T_{c}$. It is clear that the bulk viscosity effect is prominent near the transition temperature $T \approx 1.5 T_{c}$ and the drag coefficient approaches the conformal limit $\left(c_{s}^{2} \approx \frac{1}{3}\right)$ at high temperatures $T \gtrsim 4 T_{c}$. This behaviour can be explained from Eq. (20) where in the limit $T>>T_{c}$, we have $z_{k} \rightarrow 1$ (ideal equation of state) and the medium modified part of the dispersion relation vanishes, i.e., $\delta \omega_{k} \rightarrow 0$ in Eq. (12). The remaining two terms get cancelled in our case of the massless quasiparticles, leading to effectively zero contribution from $\delta f_{k}^{\text {bulk }}$ at high temperature. We also notice that both collisional and radiative processes show identical behaviour with the variation in bulk viscosity.

\section{Conclusion}

We have investigated the transport coefficients of the heavy (charm) quark in the viscous QGP by considering its Brownian motion in the hot QCD medium using the Fokker-Planck dynamics. The inelastic process of soft gluon radiation by the heavy quark is studied along with the elastic collision interactions with the medium constituents. The thermal medium interactions are included in the analysis through EQPM effective degrees of freedom. The shear and bulk viscous corrections are incorporated at leading order into the quarks, antiquarks, and gluon momentum distribution functions which are obtained by solving the effective Boltzmann equation based on the EQPM framework. The viscous corrections to the charm quark drag and diffusion coefficients have been estimated as a function of its initial momentum and QGP temperature. We observe that the shear 
viscous correction to the heavy quark transport coefficients is prominent for low momentum as well as near the transition temperature. The preliminary result for leading order bulk viscous correction is also presented for the charm quark drag coefficient. In future, we plan to include the shear and bulk viscous corrections up to the second-order into the in-medium particle distribution function.

\section{Acknowledgments}

A.S. thank the organizers of CHARM2020 workshop for the opportunity to present this work.

\section{References}

[1] A. Andronic et al., Heavy-flavour and quarkonium production in the LHC era: from proton-proton to heavy-ion collisions, Eur. Phys. J. C 76, 107 (2016).

[2] F. Prino and R. Rapp, Open heavy flavor in QCD matter and in nuclear collisions, J. Phys. G 43, 093002 (2016).

[3] A. Aarts et al., Heavy-flavor production and medium properties in high-energy nuclear collisions -What next?, Eur. Phys. J. A 53, 93 (2017).

[4] A. Beraudo et al., Extraction of heavy-flavor transport coefficients in QCD matter, Nucl. Phys. A 979, 21 (2018).

[5] X. Dong and V. Greco, Heavy quark production and properties of Quark-Gluon Plasma, Prog. Part. Nucl. Phys. 104, 97 (2019).

[6] B. Svetitsky, Diffusion of charmed quarks in the quark-gluon plasma, Phys. Rev. D 37, 2484 (1988).

[7] M. G. Mustafa, D. Pal, and D. K. Srivastava, Propagation of charm quarks in equilibrating quark - gluon plasma, Phys. Rev. C 57, 889 (1998).

[8] S. K. Das, J. e. Alam, and P. Mohanty, Probing quark gluon plasma properties by heavy flavors, Phys. Rev. C 80, 054916 (2009).

[9] S. K. Das, V. Chandra, and J. Alam, Heavy-quark transport coefficients in a hot viscous quark-gluon plasma medium, J. Phys. G 41, 015102 (2014).

[10] T. Song, P. Moreau, J. Aichelin, and E. Bratkovskaya, Exploring non-equilibrium quark-gluon plasma effects on charm transport coefficients, Phys. Rev. C 101, 044901 (2020).

[11] B. Singh and H. Mishra, Heavy quark transport in a viscous semi-QGP, Phys. Rev. D 101, 054027 (2020).

[12] M. Kurian, V. Chandra, and S. K. Das, Impact of longitudinal bulk viscous effects to heavy quark transport in a strongly magnetized hot QCD medium, Phys. Rev. D 101, 094024 (2020). 
[13] M. Kurian, M. Singh, V. Chandra, S. Jeon, and C. Gale, Charm quark dynamics in quark-gluon plasma with 3+1D viscous hydrodynamics, Phys. Rev. C 102, 044907 (2020).

[14] V. Chandra and V. Ravishankar, A quasi-particle description of $(2+1)$ - flavor lattice QCD equation of state, Phys. Rev. D 84, 074013 (2011).

[15] V. Chandra and V. Ravishankar, Quasi-particle model for lattice QCD: Quark-gluon plasma in heavy ion collisions, Eur. Phys. J. C 64, 63 (2009).

[16] S. Mitra and V. Chandra, Covariant kinetic theory for effective fugacity quasiparticle model and first order transport coefficients for hot QCD matter, Phys. Rev. D 97, 034032 (2018).

[17] A. Shaikh, M. Kurian, S. K. Das, V. Chandra, S. Dash, and B. K. Nandi, Heavy quark transport coefficients in a viscous QCD medium with collisional and radiative processes, Phys. Rev. D 104, 034017 (2021).

[18] S. Mazumder, T. Bhattacharyya, and J. Alam, Gluon bremsstrahlung by heavy quarks - its effects on transport coefficients and equilibrium distribution, Phys. Rev. D 89, 014002 (2014).

[19] X.-N. Wang, M. Gyulassy, and M. Plumer, The LPM effect in QCD and radiative energy loss in a quark gluon plasma, Phys. Rev. D 51, 3436 (1995).

[20] R. Abir, C. Greiner, M. Martinez, M. G. Mustafa, and J. Uphoff, Soft gluon emission off a heavy quark revisited, Phys. Rev. D 85, 054012 (2012).

[21] S. Mitra and V. Chandra, Transport coefficients of a hot QCD medium and their relative significance in heavy-ion collisions, Phys. Rev. D 96, 094003 (2017).

[22] A. Jaiswal, Relativistic dissipative hydrodynamics from kinetic theory with relaxation time approximation, Phys. Rev. C 87, 051901 (2013).

[23] S. Bhadury, M. Kurian, V. Chandra, and A. Jaiswal, First order dissipative hydrodynamics and viscous corrections to the entropy four-current from an effective covariant kinetic theory, J. Phys. G 47, 085108 (2020).

[24] J. D. Bjorken, Highly Relativistic Nucleus-Nucleus Collisions: The Central Rapidity Region, Phys. Rev. D 27, 140 (1983). 